\title{
Correction: Mesenchymal stem cell-derived exosomal miR-223 regulates neuronal cell apoptosis
}

Hong Wei, Yuhao Xu, Qi Chen, Hui Chen, Xiaolan Zhu and Yuefeng Li

Correction to: Cell Death and Disease

https://doi.org/10.1038/s41419-020-2490-4

published online 27 April 2020
The original version of this article did not acknowledge Yuefeng $\mathrm{Li}$ as a corresponding author. This has now been corrected in both the PDF and HTML versions of the article.

Published online: 08 June 2020 